\section{VTE: Neuer Score zeigt an, wer keine Langzeit-Antikoagulation benötigt}

\author{
Ein 4-Punkte-Score erfasst zuverlässig Patientinnen mit niedrigem Rezidiv- \\ Risiko nach venösen Thromboembolien. Der Hälfte aller betroffenen Frauen \\ kann so die langfristige Antikoagulation erspart werden.
}

$\mathrm{N}$ ach einer ersten Episode einer venösen Thromboembolie (VTE), für die kein Auslöser identifiziert wird, lautet die Leitlinien-Empfehlung langfristig Antikoagulation. Dies scheint zumindest für einen Teil der Patienten eine Übertherapie darzustellen. Mithilfe der sog. HERDOO2-Regel, die vier wesentlichen Risikofaktoren erfasst, können Patientinnen mit niedrigem Risiko identifiziert werden, die auf die Antikoagulation verzichten können. Für Männer ist die Regel jedoch nicht anwendbar.

Bei den vier Risikofaktoren handelt es sich um:

1. Hyperpigmentation, Ödem oder Rötung in einem Bein,

2. D-Dimer $>150 \mu \mathrm{g} / \mathrm{ml}$ unter Antikoagulation,

3.Adipositas mit einem BMI $>30 \mathrm{~kg} / \mathrm{m}^{2}$

4.Alter über 65 Jahre,
Die ersten Buchstaben der englischen Übersetzung dieser Faktoren ergeben das bizarre Wort HERDOO.

In der REVERSE II-Studie wurde international multizentrisch bei $2.779 \mathrm{~Pa}$ tienten mit erster nicht-provozierter VTE überprüft, ob sich mithilfe der HERDOO2-Regel Frauen identifizieren lassen, die ein jährliches Rezidiv-Risiko von unter $5 \%$ aufweisen und die Antikoagulation daher beenden können.

Wie die Autoren um Dr. Marc Roger, Universitätsklinik in Ottawa, beim ESC berichteten, wurden alle Patienten zunächst fünf bis zwölf Monate lang mit Antikoagulanzien behandelt. Anschließend wurde das Rezidiv-Risiko der Frauen mit der HERDOO2-Regel bestimmt: 0-1 Punkte definierten niedriges, 2 und mehr Punkte hohes Risiko. Bei den meisten der 622 Frauen mit niedrigem Risiko wurde die Antikoagulation abgesetzt. Im nächsten Jahr lag ihr VTE-Rezidiv-Risiko bei $3 \%$. Frauen in dieser Gruppe, die die Antikoagulation fortsetzten, hatten zu $0 \%$ Rezidive.

Die meisten der 591 Frauen mit hohem Risiko, und ebenso alle Männer, setzten die Antikoagulation fort. Ihr Rezidiv-Risiko betrug 1,6\% im Folgejahr. 323 Frauen und Männer der HochrisikoGruppe stoppten die Antikoagulation; $8,1 \%$ von ihnen erlitten im nächsten Jahr ein Rezidiv.

Die Autoren folgern daraus, dass mit der HERDOO2-Regel bei etwa $50 \%$ aller Patientinnen eine geringe RezidivGefahr ermittelt werden kann. Das Restrezidiv-Risiko von $3 \%$ halten sie für niedrig genug, um ein Absetzen zu empfehlen; zumal die Antikoagulation mit einer Blutungsgefahr einhergeht.

Womöglich lässt sich die Regel durch Adjustierung der Altersgrenze weiter verfeinern. In der Subgruppe der postmenopausalen Frauen mit HERDOO2Score von $0-1$, die älter als 50 Jahre alt waren, erlitten 5,7\% im Jahr nach dem Absetzen ein Rezidiv.

Dr. med. Dirk Einecke

märe Thrombolyse-Behandlung, an die sich die Thrombektomie anschloss.

\section{Gleiche Ergebnisse}

In der ersten Gruppe betrug die mediane Zeit zwischen Schlaganfall und Thrombektomie 147 Minuten, in der zweiten Gruppe 188 Minuten. Dennoch waren die Ergebnisse gleich, 41 \% der Patienten erzielten in beiden Gruppen gute funktionelle Ergebnisse. Die Rate ist etwas geringer als die $48 \%$, die im Schnitt in den 2015 veröffentlichten randomisierten Studien in spezialisierten neuroradiologischen Abteilungen erreicht wurden.

Ebenso deutet die Studie an, dass die Therapie auch interdisziplinär in interventionellen kardiologischen Labors erfolgen kann, deren Dichte meist höher ist als jener spezialisierter NeurologieEinrichtungen. Jedoch sollten die Ergebnisse in größeren Studien überprüft werden. Dr. med. Dirk Einecke 